\title{
Cerebral Venous Thrombosis: MR Black-Blood Thrombus Imaging with Enhanced Blood Signal Suppression
}

\author{
(D) G. Wang, (D)X. Yang, (D). Duan, (D) N. Zhang, (D) M.M. Maya, (D) Y. Xie, (D)X. Bi, (D)X. Ji, (DD. Li, (D) Q. Yang, and (D). Fan
} \& $\mathrm{O} \equiv$

\begin{abstract}
BACKGROUND AND PURPOSE: The residual blood flow artifact is a critical confounder for MR black-blood thrombus imaging of cerebral venous sinus thrombosis. This study aimed to conduct a validation of a new MR black-blood thrombus imaging technique with enhanced blood signal suppression.
\end{abstract}

\begin{abstract}
MATERIALS AND METHODS: Twenty-six participants (13 volunteers and 13 patients) underwent conventional imaging methods followed by 2 randomized black-blood thrombus imaging scans, with a preoptimized delay alternating with nutation for tailored excitation (DANTE) preparation switched on and off, respectively. The signal intensity of residual blood, thrombus, brain parenchyma, normal lumen, and noise on black-blood thrombus images were measured. The thrombus volume, SNR of residual blood, and contrast-to-noise ratio for residual blood versus normal lumen, thrombus versus residual blood, and brain parenchyma versus normal lumen were compared between the 2 black-blood thrombus imaging techniques. Segmental diagnosis of venous sinus thrombosis was evaluated for each black-blood thrombus imaging technique using a combination of conventional imaging techniques as a reference.
\end{abstract}

RESULTS: In the volunteer group, the SNR of residual blood (11.3 \pm 2.9 versus $54.0 \pm 23.4, P<.001)$ and residual blood-to-normal lumen contrast-to-noise ratio $(7.5 \pm 3.4$ versus $49.2 \pm 23.3, P<.001)$ were significantly reduced using the DANTE preparation. In the patient group, the SNR of residual blood (16.4 \pm 8.0 versus $75.0 \pm 35.1, P=.002)$ and residual blood-to-normal lumen contrast-to-noise ratio $(12.4 \pm 7.8$ versus $68.8 \pm 35.4, P=.002)$ were also significantly lower on DANTE-prepared black-blood thrombus imaging. The new black-blood thrombus imaging technique provided higher thrombus-to-residual blood contrast-to-noise ratio, significantly lower thrombus volume, and substantially improved diagnostic specificity and agreement with conventional imaging methods.

CONCLUSIONS: DANTE-prepared black-blood thrombus imaging is a reliable MR imaging technique for diagnosing cerebral venous sinus thrombosis.

ABBREVIATIONS: BTI = black-blood thrombus imaging; $\mathrm{CE}=$ contrast-enhanced; $\mathrm{CNR}=$ contrast-to-noise ratio; CVT = cerebral venous sinus thrombosis; DANTE = delay alternating with nutation for tailored excitation; TSE = turbo spin-echo; SPACE = sampling perfection with application-optimized contrasts by using different flip angle evolution

erebral venous sinus thrombosis (CVT) is a potentially lifethreatening cerebrovascular disorder that most often affects young individuals. ${ }^{1} \mathrm{MR}$ imaging is currently the best noninvasive imaging technique for the diagnosis of CVT. ${ }^{2}$ A series of MR images, such as T1- and T2-weighted turbo spin-echo (TSE),

Received May 10, 2019; accepted after revision July 29

From the Department of Biomedical Sciences (G.W., N.Z., Y.X., D.L., Q.Y., Z.F.), Biomedical Imaging Research Institute, and Department of Imaging (M.M.M.), Cedars-Sinai Medical Center, Los Angeles, California; Department of Radiology (G.W.), The First Affiliated Hospital of China Medical University, Shenyang, China; Departments of Emergency (J.D., X.J.) and Radiology (X.Y., Q.Y.) Xuanwu Hospital, Capital Medical University, Beijing, China; Paul C. Lauterbur Research Center for Biomedical Imaging (N.Z.), Shenzhen Institutes of Advanced Technology, Chinese Academy of Sciences, Shenzhen, China; MR R\&D (X.B., D.L.), Siemens Healthineers, Los Angeles, California; and Departments of Medicine (D.L., Z.F.) and Bioengineering (Z.F.), University of California, Los Angeles, California. G. Wang and X. Yang have contributed equally to this work.
$\mathrm{T} 2{ }^{*}$-weighted gradient recalled-echo, and MRV, are usually combined to confirm the diagnosis and stage of CVT. However, technical limitations associated with each of these methods may

This work was supported by National Institutes of Health/National Heart, Lung, and Blood Institute 1 R01 HL147355 and Beijing Natural Science Foundation (No. 17L20253), The National Science Foundation of China (No. 91749127 and 81830056), and Beijing Municipal Administration of Hospitals Clinical Medicine Development of Special Funding Support. (ZYLX201706).

Please address correspondence to Zhaoyang Fan, PhD and Qi Yang, MD, PhD, 8700 Beverly Blvd, Pacific Theatres Suite 400, Los Angeles, CA 90048, e-mail: Zhaoyang.Fan@csmc.edu; yangyangqiqi@gmail.com

\footnotetext{
- Indicates open access to non-subscribers at www.ajnr.org

$\equiv$ Indicates article with supplemental on-line appendix. Indicates article with supplemental on-line photos.

http://dx.doi.org/10.3174/ajnr.A6212
} 
result in an equivocal diagnosis. ${ }^{3}$ Moreover, inconsistent spatial coverage and resolution among these $2 \mathrm{D}$ and $3 \mathrm{D}$ scans along with their potential misregistration would preclude precise characterization of CVT in, for example, its location, extent, and the degree of recanalization. Detailed knowledge of these aspects is, however, relevant to treatment decision-making and therapeutic response monitoring. ${ }^{4,5}$

Recently, an MR black-blood thrombus imaging (BTI) method based on a T1-weighted 3D variable-flip angle TSE sequence was proposed for early detection of CVT. 6 The sequence has an inherent black-blood effect and superior SNR performance. ${ }^{7}$ The black-blood image contrast in BTI allows thrombi to be visually isolated within the dark lumen of the venous sinuses. Configured as a T1-weighted acquisition, BTI is particularly useful for the detection of subacute CVT, which is rich in short-T1 methemoglobins and thus appears hyperintense with respect to surrounding brain parenchyma and sinus lumen.

Despite the high sensitivity and specificity reported for the detection of subacute CVT, ${ }^{6}$ BTI is potentially limited in accurately characterizing acute (particularly hyperacute) or chronic CVT due to the presence of residual blood flow artifacts. Residual signal intensity was observed in the venous sinuses, which has been attributed to slow flow velocity and inadequate blood signal attenuation of the sequence. ${ }^{6}$ While such artifacts have a negligible influence on the detection of subacute CVT, they may pose a diagnostic challenge in the detection of thrombi in chronic and acute CVT because thrombi and venous blood have similar signal intensities on T1-weighted images. ${ }^{8}$

The goal of this work was to present an MR BTI technique with enhanced blood signal attenuation and perform a clinical study to validate its improved performance in imaging of CVT and to demonstrate the feasibility of CVT characterization.

\section{MATERIALS AND METHODS Study Population}

Twenty-six participants, including 13 healthy volunteers (9 women; 35-65 years of age; mean age, 53 years) and 13 patients (7 women; $19-54$ years of age; mean age, 35 years), were recruited to undergo MR BTI studies. The inclusion criteria were no history of cerebrovascular diseases for healthy controls and CVT diagnosed on the basis of clinical symptoms and conventional imaging techniques (CT, TSE, SWI, contrast-enhanced [CE]MRV, and TOF-MRV) for patients. Exclusion criteria included contraindications for MR imaging and intolerance to additonal MR images. Xuanwu Hospital institutional review committee approval and patients' informed consent were obtained.

\section{Imaging System}

Imaging was performed on a 3T whole-body system (Magnetom Verio; Siemens, Erlangen, Germany). A standard head-neck 12channel coil was used for receiving signals from a volume ranging from the superior sagittal sinus to the internal jugular veins.

\section{MR Imaging Sequence}

The sequence used for MR BTI was designed on the basis of sampling perfection with application-optimized contrasts by using different flip angle evolution (SPACE sequence; Siemens). ${ }^{7}$ A previously proposed black-blood preparation method, delay alternating with nutation for tailored excitation (DANTE), ${ }^{9}$ was used to improve the suppression of venous flow signals while introducing minimal T2 weighting. ${ }^{10,11}$ The DANTE module consists of a train of short, hard radiofrequency pulses interspersed with dephasing gradients that are applied simultaneously in all 3 orthogonal directions. The module is followed by chemically selective fat saturation and a T1-weighted SPACE readout. Whole-head spatial coverage is achieved using nonselective hard radiofrequency pulses for excitation, which averts the need for multiple signal averages to suppress free-induction-decay artifacts. ${ }^{7}$ A saggitally oriented imaging volume is prescribed, which requires fewer partitions than other imaging orientations, to further reduce the imaging time. Three 40-mm-wide spatial presaturation bands are applied immediately before excitation to suppress the signals from the nose and ears if they are located outside the prescribed imaging volume. The parameters of the DANTE preparation were optimized for adequate flow signal suppression in a separate volunteer study (detailed in the On-line Appendix).

\section{Imaging Protocol}

All participants underwent conventional MR imaging sequences followed by 2 randomized BTI scans, with DANTE preparation switched on (DANTE+) and off (DANTE-), respectively. The major imaging parameters used in the SPACE readout were the following: $\mathrm{TR} / \mathrm{TE}=600 / 14 \mathrm{~ms} ; \mathrm{FOV}=300 \times 206 \times 162 \mathrm{~mm}^{3}$; matrix size $=384 \times 264 \times 208$; spatial resolution $=0.78 \mathrm{~mm}$ isotropic; 6/8 partial Fourier in the partition-encoding direction; echo-train length $=36$; parallel imaging (generalized autocalibrating partially parallel acquisition) acceleration factor $=2$ in the phase-encoding direction; elliptic $k$-space sampling; scan time $=$ 5 minutes 40 seconds.

\section{Image Analysis}

Image review and signal intensity measurements were performed on a workstation (syngo MultiModality Workplace; Siemens), where multiplanar reformation, maximum intensity projection, and minimum intensity projection functionalities were available to image reviewers. The venous system was divided into the following 16 segments: superior sagittal sinus, inferior sagittal sinus, straight sinus, confluence of sinuses, right transverse sinus, left transverse sinus, right sigmoid sinus, left sigmoid sinus, vein of Galen, internal cerebral vein, basal vein of Rosenthal, vein of Labbé, right cortical vein, left cortical vein, right internal jugular vein, and left internal jugular vein.

For the healthy volunteer group, both BTI DANTE+ and DANTE - image sets of each subject were reviewed side by side by a radiologist (G.W.) with 8 years of experience in MR image interpretation to identify residual blood signals in individual segments. When residual blood was observed in a segment, mean signal intensity was measured. The mean signal intensity of the adjacent brain parenchyma and normal lumen and noise $(\sigma, \mathrm{SD}$ of signal intensity in the adjacent air space) were also measured. The contrast-to-noise ratio $(\mathrm{CNR})\left(\mathrm{A}\right.$-to-B CNR $\left.=\left[\mathrm{SI}_{\mathrm{A}}-S I_{\mathrm{B}}\right] / \sigma\right)$, where SI indicates signal intensity, was calculated for residual blood versus normal lumen (indicating the blood-suppressing 
performance of DANTE) and for brain parenchyma versus normal lumen (indicating the overall sacrifice in the black-blood contrast caused by DANTE), respectively.

For the patient group, 2 radiologists with 13 years (Q.Y.) and 8 years (G.W.) of experience in MR image interpretation, respectively, performed consensus reading. Combined conventional sequences from the 13 subjects were blindly reviewed for segment-level diagnosis of CVT. One month later, diagnostic review was also performed on the randomized 26 BTI image sets (2 sets per subject). Visible signals within the dark sinus lumens were deemed "apparent thrombi," unless they were of granule shape (highly indicative of arachnoid granulations) or appeared as floating patches (highly indicative of residual blood rather than thrombi). Furthermore, the total volume of apparent thrombi was quantified in the superior sagittal sinus by manually contouring the thrombi section by section using commercial software (Vessel Analysis, Beijing Sirui Star Technology Co., Ltd.).

The 2 BTI image sets from each patient were then reviewed side by side to scrutinize the actual incidence of residual blood and true thrombus by the same 2 radiologists. The finalized true thrombi were individually categorized into hyperintense, isointense, or hybrid types on the basis of their appearance

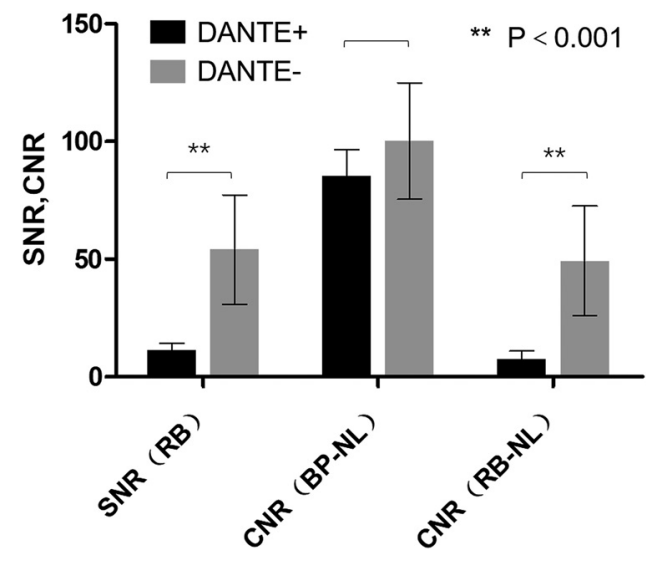

FIG 1. SNR of residual blood (RB), CNR between brain parenchyma (BP) and normal lumen (NL), and CNR between RB and NL in the healthy volunteer group. SNR of RB and RB-to-NL CNR were significantly reduced on BTI DANTE + images compared with those on BTI DANTE- images. Double asterisks denote $P<.001$. with respect to adjacent normal brain parenchyma. Signal intensity measurement was performed, respectively, for the regions of residual blood and residual blood nearest isointense and hyperintense thrombus, normal lumen, and brain parenchyma on both image sets. The CNRs of residual blood or thrombus to other tissues were calculated.

\section{Statistical Analysis}

Statistical analysis was performed using SPSS (Version 16.0; IBM, Armonk, New York). A 2-tailed Wilcoxon signed rank test was used to determine the difference in the thrombus volume, SNR, and CNR between the 2 BTI techniques. A Cohen $\kappa$ test was used to determine the agreement in the diagnosis of CVT at the per-segment level between BTI and conventional imaging techniques. Conventional imaging techniques were used as the reference standard for assessing the sensitivity, specificity, and negative and positive predictive values of BTI techniques. Statistical significance was defined as $P<.05$.

\section{RESULTS}

All 26 participants successfully underwent both BTI scans. In the volunteer group, the SNR of residual blood $(11.3 \pm 2.9$ versus $54.0 \pm 23.4, P<.001)$ and residual blood-to-normal lumen CNR $(7.5 \pm 3.4$ versus $49.2 \pm 23.3, P<.001)$ were significantly reduced on BTI DANTE + images compared with BTI DANTEimages, whereas there was no significant difference in brain parenchyma-to-normal lumen $\mathrm{CNR}(85.4 \pm 11.2$ versus $100.2 \pm$ 24.7, $P=.060$ (Fig 1).

The clinical characteristics of patients are listed in Table 1. Two patients were clinically diagnosed with acute CVT (0-7 days after symptom onset); 2 patients, with subacute CVT (7-14 days); and 9 patients, with chronic CVT ( $\geq 15$ days). CVT was detected in 11 subjects and 72 segments on conventional images. Blinded review of BTI reported apparent thrombi in 11 subjects and 77 segments on BTI DANTE+ but in 13 subjects and 94 segments on BTI DANTE- (Table 2). The agreement at the per-segment level between BTI and conventional imaging techniques was excellent for BTI DANTE $+(\kappa=0.964)$ but moderate for BTI DANTE- $(\kappa=0.770)$. The specificities of CVT detection at the per-segment level were $96.3 \%$ and $83.8 \%$, respectively, for the 2 different BTI techniques (Table 3). Representative cases are shown in Fig 2. In the 9 patients who were diagnosed with apparent thrombi in the superior sagittal sinus, the thrombus

Table 1: Clinical characteristics of patients with CVT

\begin{tabular}{|c|c|c|c|c|c|}
\hline Patient & Sex & Age (yr) & Symptom & Duration & Conventional Imaging Methods \\
\hline 1 & $\mathrm{~F}$ & 47 & Headache & 2 days & TSE, SWI, CE-MRV, TOF-MRV, CT \\
\hline 2 & M & 19 & Headache & 10 days & TSE, TOF-MRV, CT \\
\hline 3 & M & 54 & Focal neurologic deficit & 13 days & TSE, SWI, CE-MRV, TOF-MRV, CT \\
\hline 4 & M & 30 & Headache, focal neurological deficit & 20 days & TSE, CE-MRV, TOF-MRV, CT \\
\hline 5 & M & 44 & Seizures & 25 days & TSE, TOF-MRV \\
\hline 6 & $\mathrm{~F}$ & 53 & Headache & 27 days & TSE, SWI, CE-MRV, TOF-MRV, CT \\
\hline 7 & M & 42 & Headache & $1 \mathrm{mo}$ & TSE, CE-MRV, TOF-MRV \\
\hline 8 & $\mathrm{~F}$ & 32 & Headache & $1 \mathrm{mo}+22$ days & TSE, SWI, CE-MRV, TOF-MRV, CT \\
\hline 9 & M & 19 & Headache & $2 \mathrm{mo}$ & TSE, TOF-MRV, CT \\
\hline 10 & $\mathrm{~F}$ & 28 & Headache & $4 \mathrm{mo}$ & TSE, TOF-MRV, CT \\
\hline 11 & $\mathrm{~F}$ & 45 & Headache, papilledema & $4 \mathrm{mo}$ & SWI, TOF-MRV, CT \\
\hline 12 & $\mathrm{~F}$ & 36 & Headache & 1 year & TSE, CE-MRV, TOF-MRV, CT \\
\hline 13 & $\mathrm{~F}$ & 19 & Headache & 2 days & TSE, TOF-MRV, CE-MRV \\
\hline
\end{tabular}


Table 2: Locations of thrombi and residual flow artifacts identified on BTI with and without DANTE preparation ${ }^{\text {a }}$

\begin{tabular}{|c|c|c|c|c|c|c|c|c|c|c|c|c|c|c|c|c|}
\hline \multirow[b]{2}{*}{ Pt. No. } & \multicolumn{16}{|c|}{ Segments } \\
\hline & SSS & ISS & VG & SS & CS & RTS & LTS & RSS & LSS & ICV & BVR & VL & $\mathrm{RC}$ & LC & RJV & LJV \\
\hline 1 & $=$ & & & & $=$ & & $=$ & & & & & & $=$ & $=$ & & \\
\hline 2 & & & & & & $=$ & & $=$ & & & & & & & & \\
\hline 3 & $+/=$ & & $=$ & & + & $=$ & $=$ & & & & & & & + & + & \\
\hline 4 & $=$ & & $=$ & $=$ & $+/=$ & $=$ & $=$ & + & $=$ & & & & $=$ & $=$ & $=$ & \\
\hline 5 & & & & & & & & & & & & & & & & \\
\hline 6 & $=$ & & $=$ & & & $=$ & $=$ & $=$ & $=$ & & & & $=$ & $=$ & & \\
\hline 7 & $=$ & & & & $=$ & $=$ & $=$ & $+/=$ & & & & & $=$ & $=$ & & \\
\hline 8 & $+/=$ & & + & $+/=$ & $+/=$ & $+/=$ & $=$ & $=$ & $=$ & $=$ & + & & & & $+/=$ & $+/=$ \\
\hline 9 & $=$ & $=$ & & & $=$ & $=$ & $=$ & $=$ & $=$ & & & & & $=$ & $=$ & \\
\hline 10 & $+/=$ & & & $+/=$ & $+/=$ & & $+/=$ & & $+/=$ & & & & & & & $=$ \\
\hline 11 & & & & & & & & & & & & & & & & \\
\hline 12 & $=$ & & & & $=$ & $=$ & $=$ & $=$ & $=$ & & & & & & & $=$ \\
\hline 13 & & & & & & & $=$ & & $=$ & & & & & & & $=$ \\
\hline
\end{tabular}

Note:-SSS indicates superior sagittal sinus; ISS, inferior sagittal sinus; VG, vein of Galen; SS, straight sinus; CS, confluence of sinus; RTS, right transverse sinus; LTS, left transverse sinus; RSS, right sigmoid sinus; LSS, left sigmoid sinus; ICV, internal cerebral vein; BVR, basal vein of Rosenthal; VL, vein of Labbé; RC, right cortical vein; LC, left cortical vein; RJV, right jugular vein; LJV, left jugular vein; =, iso-intense thrombus; + , hyperintense thrombus; $+/=$, hybrid thrombus.

${ }^{a}$ Shadow indicates that residual flow artifacts are present in the segment on BTI without DANTE preparation but not on BTI with DANTE preparation.

volume was significantly lower $(4.814 \pm 2.278 \mathrm{~mL}$ versus $6.341 \pm 2.302 \mathrm{~mL}, P=.008)$ when using the DANTE preparation (Fig 3).

Table 3: Diagnostic performance of BTI with/without DANTE for the detection of CVT on the per-segment level

\begin{tabular}{lcc}
\hline & Dante + & Dante- \\
\hline Sensitivity & $100 \%$ & $100 \%$ \\
Specificity & $96.3 \%$ & $83.8 \%$ \\
PPV & $93.5 \%$ & $76.6 \%$ \\
NPV & $100 \%$ & $100 \%$ \\
FP & $3.7 \%$ & $16.2 \%$ \\
\hline
\end{tabular}

Note:-PPV indicates positive predictive value; NPV, negative predictive value; FP, false-positive.
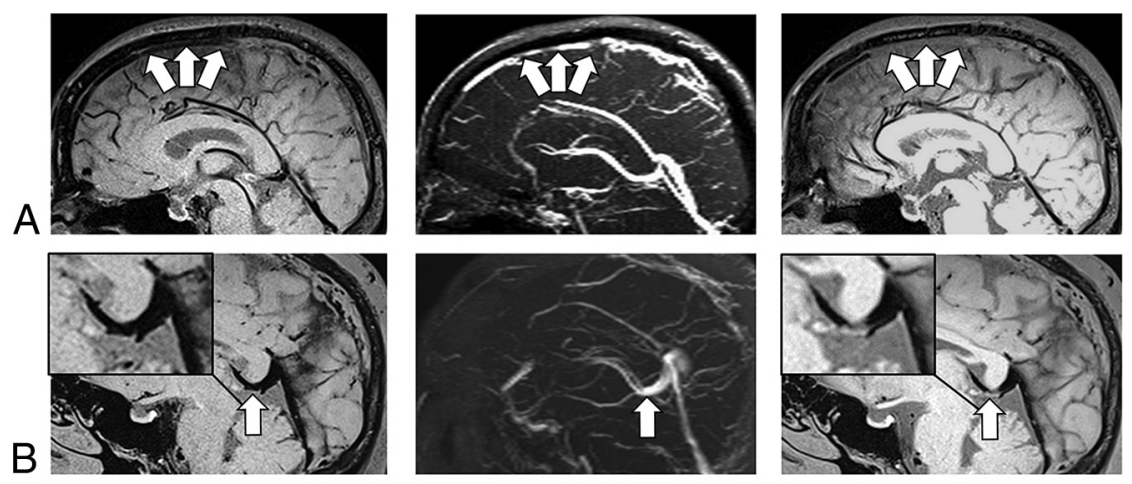

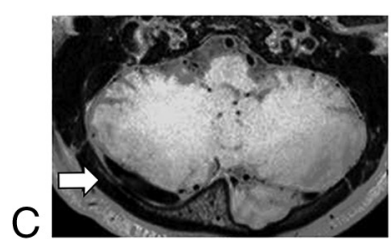

DANTE+

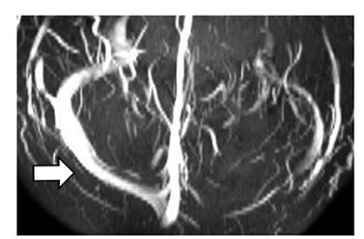

MRV

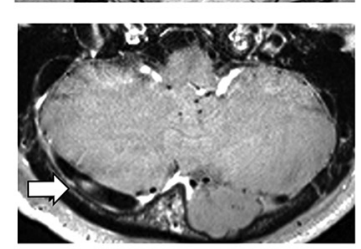

DANTE-
FIG 2. BTI (with/without DANTE preparation) images and MRV images of 3 patients with CVT. On BTI DANTE - images, isointense signals appear in the superior sagittal sinus (arrows, A), vein of Galen (arrow, $B$ ), and right transvers sinus (arrow, $C$ ). However, they are not shown on the BTI DANTE+ images (arrows). MRVs for these 3 patients demonstrate no filling defects on corresponding segments (arrows).
Residual blood signals in patients with CVT were also dramatically suppressed using the DANTE preparation. The SNR of residual blood $(16.4 \pm 8.0$ versus $75.0 \pm 35.1, P=.002)$ and residual blood-to-normal lumen CNR $(12.4 \pm 7.8$ versus $68.8 \pm 35.4, \quad P=.002)$ were significantly reduced on BTI DANTE + . Furthermore, the thrombus-to-residual blood CNR preparation had negligible impact on the contrast between thrombi and surrounding tissues (Fig 4B).

\section{DISCUSSION}

Our work presents a further refinement in blood signal suppression for MR BTI. This is the first study to evaluate the effectiveness and clinical practicability of BTI with the DANTE preparation in healthy subjects and those with CVT, to our knowledge. Our initial results demonstrate improved performance of the technique in the detection of thrombi and the feasibility of quantifying the thrombus volume and assessing the degree of recanalization.

Blood signal suppression is the most important contributing factor for the improved accuracy of MR BTI in the detection of CVT, regardless of its stage. While the SPACE sequence used in the original BTI technique has inherent black-blood contrast, residual blood flow signals were observed here in both healthy subjects and patients. This suggests 
that its blood-suppressing capacity is insufficient for slow or even stagnant venous blood flow in the sinuses. Such image artifacts can mimic thrombus (isointense), resulting in falsepositives or overestimation of CVT. Additional blood signal suppression is therefore indispensable. Previous work has reported that the DANTE preparation is sensitive to blood flow over a broad range of velocities above approximately $1 \mathrm{~mm} / \mathrm{s}^{9}$ In our study, this approach demonstrated the effectiveness of eliminating residual flow signals as evidenced by significantly reduced SNR of residual blood and residual blood-to-normal lumen CNR compared with that measured from the original BTI. On the other hand, this approach did not substantially sacrifice the CNR of thrombi to the normal sinus lumen and brain parenchyma, indicating its

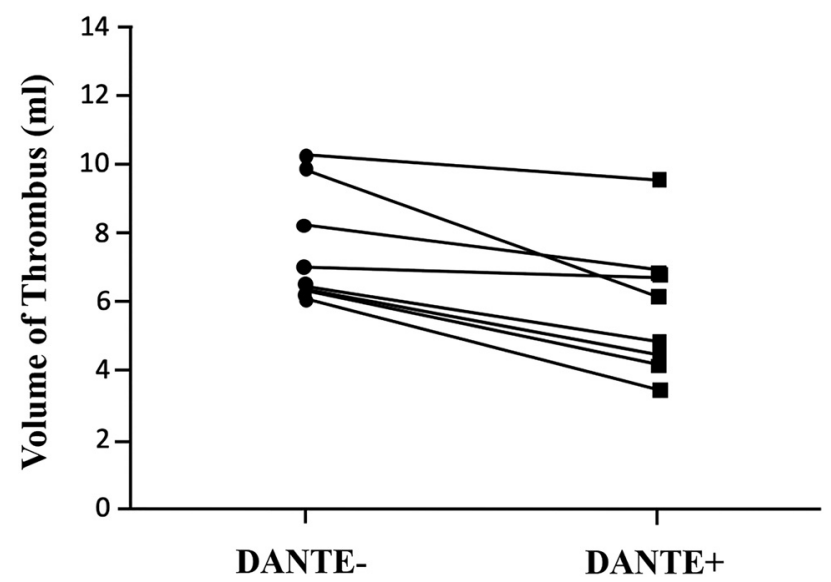

FIG 3. Thrombus volumes on BTI DANTE + and BTI DANTE- images. In the 8 patients who were diagnosed with apparent thrombi in the superior sagittal sinus, the measured thrombus volume was significantly lowered $(4.814 \pm 2.278 \mathrm{~mL}$ versus $6.341 \pm 2.302 \mathrm{~mL}$, $P=.008)$ when using the DANTE preparation. minimal effect on static tissues. As a result, the diagnosis of CVT was remarkably improved, with combined conventional imaging techniques as the reference.

The feasibility of quantifying thrombus volumes is shown in our study. Thrombus volume was manually measured and demonstrated significant reduction in BTI when the DANTE preparation was used. Although quantification of thrombus volume is not yet a common clinical practice, presumably due to the lack of reliable thrombus-depicting approaches, this quantitative marker could be useful for following up the effect of clinical treatment and guiding treatment decisions on dose and duration. Conventional methods such as MRV and TSE are indirect approaches by detecting filling defects, which are susceptible to many factors related to the anatomy and imaging protocol. ${ }^{12-14}$ BTI allows direct differentiation of CVT from other tissues, thus facilitating the quantification procedure. In this study, in a manual method, we demonstrated significantly reduced thrombus volume in DANTE-prepared MR BTI. In the future, MR BTI with DANTE is potentially helpful to quantitatively assess the recanalization effect of different clinical treatments as well as to compare the relationship between thrombus quantification and outcome.

The imaging protocol used in this study also incorporates additional features that will facilitate the translation of MR BTI into clinical practice. Specifically, a whole-brain imaging volume with isotropic high spatial resolution can be acquired within $<6$ minutes. This is advantageous over the protocol presented by Yang et al, ${ }^{6}$ whereby spatial coverage and spatial resolution were compromised to maintain the reasonably short scan time. Moreover, a standard head-neck coil is used so that jugular veins can also be examined along with the cerebral venous system within the same scan. With these features combined, MR BTI could readily be integrated into the diagnostic work-up.

There were several limitations in the present study. First, this was a single-center study with a relatively small sample size. A multicenter trial with a large patient cohort is desirable, though the recruitment is relatively difficult because CVT is a rare disease with life-threatening potential. Second, MRV is currently considered the noninvasive test of choice for the evaluation of the dural sinus. CE-MRV (relative to time-of-flight MRV) more accurately depicts the degree of patency of thrombosed segments, ${ }^{3,15}$ though not all patients in our study underwent CE-MRV. However, flow-related and susceptibility artifacts as well as the bolus-timing issues can impair the evaluation of the venous structures. The more invasive arterial DSA is still the standard of reference but is not routinely performed in our institution. ${ }^{16}$
FIG 4. $A$, SNR of residual blood (RB), CNR between RB and normal lumen (NL), and CNR between $\mathrm{RB}$ and thrombus in the patient group. SNR of RB and RB-to-NL CNR was significantly reduced on BTI DANTE+ images compared with BTI DANTE - images. RB-to-thrombus CNR was significantly improved for the isointense thrombus type. $B$, CNR between thrombus and NL and CNR between thrombus and brain parenchyma (BP). When one used the DANTE preparation, the CNR between thrombus and NL or BP was not significantly sacrificed except for the CNR between isointense thrombus and NL. THRiso indicates isointense thrombus; THRhyper, hyperintense thrombus; asterisk, $P<.05$. 


\section{CONCLUSIONS}

The DANTE preparation significantly enhances the blackblood contrast in black-blood thrombus imaging, making the technique more accurate for the diagnosis of cerebral venous sinus thrombosis.

Disclosures: Zhaoyang Fan-RELATED: Grant: National Institutes of Health/ National Heart, Lung, and Blood Institute 1 R01 HL147355. Qi Yang—RELATED: Grant: Beijing Natural Science Foundation (No. 17L20253), National Science Foundation of China (No. 91749127 and 81830056), Beijing Municipal Administration of Hospitals Clinical Medicine Development of Special Funding Support (ZYLX201706). Guan Wang-RELATED: China Medical University as a visiting scholar.

\section{REFERENCES}

1. Bousser MG, Ferro JM. Cerebral venous thrombosis: an update. Lancet Neurol 2007;6:162-70 CrossRef Medline

2. Bianchi D, Maeder P, Bogousslavsky J, et al. Diagnosis of cerebral venous thrombosis with routine magnetic resonance: an update. Eur Neurol 1998;40:179-90 CrossRef Medline

3. Leach JL, Fortuna RB, Jones BV, et al. Imaging of cerebral venous thrombosis: current techniques, spectrum of findings, and diagnostic pitfalls. Radiographics 2006;26:S19 CrossRef Medline

4. Leach JL, Wolujewicz M, Strub WM. Partially recanalized chronic dural sinus thrombosis: findings on MR imaging, time-of-flight MR venography, and contrast-enhanced MR venography. AJNR Am J Neuroradiol 2007;28:782-89 Medline

5. Saposnik G, Barinagarrementeria F, Brown RD, et al. Diagnosis and management of cerebral venous thrombosis: a statement for healthcare professionals from the American Heart Association/ American Stroke Association. Stroke 2011;42:1158-92 Medline
6. Yang Q, Duan J, Fan Z, et al. Early detection and quantification of cerebral venous thrombosis by magnetic resonance black-blood thrombus imaging. Stroke 2016;47:404-09 CrossRef Medline

7. Mugler JP 3rd. Optimized three-dimensional fast-spin-echo MRI. J Magn Reson Imaging 2014;39:745-67 CrossRef Medline

8. Moody AR. Magnetic resonance direct thrombus imaging. $J$ Thromb Haemost 2003;1:1403-09 CrossRef

9. Li L, Miller KL, Jezzard P. DANTE-prepared pulse trains: a novel approach to motion-sensitized and motion-suppressed quantitative magnetic resonance imaging. Magn Reson Med 2012;68:142338 CrossRef Medline

10. Li L, Chai JT, Biasiolli L, et al. Black-blood multicontrast imaging of carotid arteries with DANTE-prepared 2D and 3D MR imaging. Radiology 2014;273:560-69 CrossRef Medline

11. Xie Y, Yang Q, Xie G, et al. Improved black-blood imaging using DANTE-SPACE for simultaneous carotid and intracranial vessel wall evaluation. Magn Reson Med 2016;75:2286-94 CrossRef Medline

12. Long B, Koyfman A, Runyon MS. Cerebral venous thrombosis: a challenging neurologic diagnosis. Emerg Med Clin North Am 2017;35:869-78

13. Poon CS, Chang JK, Swarnkar A, et al. Radiologic diagnosis of cerebral venous thrombosis: pictorial review. Am J Radiology 2007;189: S64-75 CrossRef Medline

14. Leach JL, Jones BV, Tomsick TA, et al. Normal appearance of arachnoid granulations on contrast-enhanced CT and MR of the brain: differentiation from dural sinus disease. AJNR Am J Neuroradiol 1996;17:1523-32 Medline

15. Stam J. Thrombosis of the cerebral veins and sinuses. $N$ Engl J Med 2005;352:1791-98 CrossRef Medline

16. Karthikeyan D, Vijay S, Kumar T, et al. Cerebral venous thrombosis-spectrum of CT findings. Indian Journal of Radiology and Imaging 2004;14:129-37 